\title{
Micro-air-gap based intrinsic Fabry-Perot interferometric fiber-optic sensor
}

\author{
Xiaopei Chen, Fabin Shen, Zhuang Wang, Zhenyu Huang, and Anbo Wang
}

\begin{abstract}
A simple intrinsic Fabry-Perot interferometric (IFPI) sensor is developed. The sensor is fabricated by two micro air gaps as reflective mirrors in a fiber to form a Fabry-Perot cavity. Theoretical and experimental studies of the sensor are described. Experimental results show that high resolution and high sensitivity can be achieved. Two structures of micro-air-gap-based IFPI sensors offer more applications than other IFPI sensors. (C) 2006 Optical Society of America

OCIS codes: $\quad 060.0060,060.2370,120.2230$.
\end{abstract}

\section{Introduction}

Intrinsic Fabry-Perot interferometric (IFPI) sensors are well known for their applications in harsh environment and distributed sensing. A typical IFPI sensor consists of two partial reflectors inside the fiber. The fiber between the two reflectors is used as the sensing device. It is called an all-fiber sensor because the light never leaves the fiber. Unlike extrinsic Fabry-Perot interferometric (EFPI) sensors, IFPI sensors have a relative flexible length of the FabryPerot (FP) cavity. A long FP cavity length generally makes the IFPI sensor more sensitive to the perturbations to be measured. The reflectance of the internal reflectors can be properly controlled to realize sensor multiplexing.

Compared with EFPI sensors, IFPI sensors are usually more difficult or need more expensive equipment to fabricate. So far, three main methods to fabricate the internal reflectors of IFPI sensors have been reported. Lee and Taylor ${ }^{1}$ introduced an IFPI sensor by producing a pair of thin-film internal mirrors inside a fiber. ${ }^{2} \mathrm{TiO}_{2}$ or $\mathrm{Al}$ thin films are coated on the cleaved fiber end faces by vacuum deposition, sputtering or electron-beam evaporation. Two fibers with one having a reflective coating are then fusion spliced to form an internal mirror. Tsai and $\mathrm{Lin}^{3}$

The authors are with the Bradley Department of Electrical and Computer Engineering, Center for Photonics Technology, Virginia Polytechnic Institute and State University, Blacksburg, Virginia 24061-0111. X. Chen's e-mail address is xichen@vt.edu.

Received 3 February 2006; revised 5 April 2006; accepted 27 April 2006; posted 28 April 2006 (Doc. ID 67721).

0003-6935/06/307760-07\$15.00/0

(C) 2006 Optical Society of America introduced an IFPI sensor by splicing two fibers with different core diameters. In the fabrication, the fibers need to be polished by using four different polishing films to obtain high-quality end faces. ${ }^{3}$ The FabryPerot (FP) cavity is formed between the interface of two fibers and the end face of the sensing fiber. The third type is UV-induced IFPI sensors. ${ }^{4}$ An excimer laser is used to introduce a refractive index change in the core of photosensitive fibers. Two adjacent index changed points form a FP cavity. In this paper, we demonstrate a simple process to fabricate micro-airgap-based IFPI sensors by differential wet chemical etching and fusion splicing.

\section{Sensor Fabrication}

The fabrication process of the micro-air-gap-based IFPI sensor consists of three steps: wet chemical etching, fusion splicing, and cleave. The following subsections provide a detailed description of these steps.

\section{A. Wet Chemical Etching}

Differential wet chemical etching is widely used in fabrication of fiber tips for photon-scanning tunnel microscopes. ${ }^{5-7}$ It takes advantage of different etching rates between the fiber core and the fiber cladding. Both sharpened and hollowed structures can be formed on a fiber end by using a suitable buffered acid solution. In the fabrication of our sensors, we used the buffered hydrofluoric acid (BHF), which is a mixture of hydrofluoric acid (HF, 50\% per weight), ammonium fluoride $\left(\mathrm{NH}_{4} \mathrm{~F}, 40 \%\right.$ per weight), and deionized (DI) water $\left(\mathrm{H}_{2} \mathrm{O}\right)$. The volume ratio of these components can be used to control the etching speed of different materials. The fiber used is Corning SMF-28, whose core is made out of Ge-doped silica 


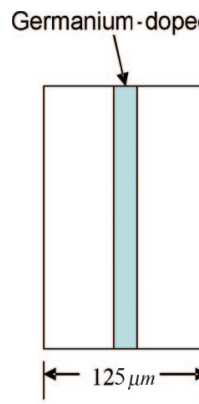

(a)

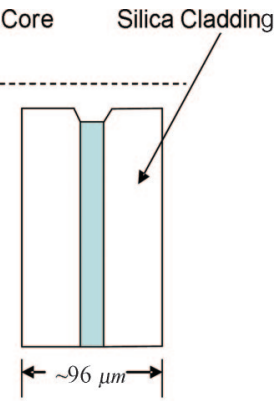

(b)

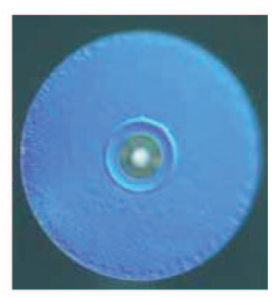

(c)
Fig. 1. (Color online) Differential wet chemical etching (Ref. 8) (a) before etching (b) after etching, (c) end face of the etched fiber.

$\left(\mathrm{SiO}_{2}+\mathrm{GeO}_{2}\right)$, and its cladding is pure silica $\left(\mathrm{SiO}_{2}\right)$. When a Ge-doped fiber is etched in BHF with a volume ratio of $\mathrm{NH} 4 \mathrm{~F}: \mathrm{HF}: \mathrm{H}_{2} \mathrm{O}=X: 1: 1$; if $X>1.7$, the core is etched faster than the cladding 5 ; therefore, a hollowed pit is formed in the center of the fiber. The differential etching process and the results are shown in Fig. 1.

Wet chemical etching is a single step process with a potential of batch fabrication. Two related issues need special attention during the sensor fabrication. The first is that the diameter of the cladding may be reduced too fast if the concentration of BHF is high. Small diameters may present difficulties to the alignment of the fiber for fusion splicing (next step). The second issue is that the etching may cause serious surface roughness. Both problems can be overcome by using the proper concentration of both $\mathrm{HN}_{4} \mathrm{~F}$ and $\mathrm{HF}$, while keeping the volume ratio of $\mathrm{HN}_{4} \mathrm{~F}$ to $\mathrm{HF}$ greater than $1.7 .{ }^{5}$

\section{B. Fusion Splicing}

The second step of sensor fabrication is to fusion splice the etched fiber with an unetched fiber. An air gap, therefore, is formed in the splice and can be used as a reflector. The fusion-splicing step is illustrated in Fig. 2 . The fusion splicing plays an important role for the control of the air-gap size. Three parameters of the splicer-fiber overlap, arc power, and arc durationdetermine the size and shape of the air gap and hence its reflectance and transmittance.

\section{Cleave}

The last step is to cleave one side of the spliced fiber to generate the second reflector as shown in Fig. 3(a). Another structure is to use two identical air gaps as reflective mirrors to form a $\mathrm{FP}$ cavity, as shown in Fig. 3(b).

In both structures, the fiber between two reflectors along with the fiber in between the function is the sensing element. The length of the fiber cavity is determined by the cleave process, which can be done under a microscope.

\section{Theoretical Analysis}

In this section, the $2 \times 2$ matrix method is used to analyze the air gap and find an optimal shape and size for our sensors. Then we apply the air-gap analysis to sensor fabrication.

The air gap is essentially a bubble inserted between two fibers. For the purpose of observation, we made a very big air gap so that it can be clearly observed under a microscope, as shown in Fig. 4(a). Based on this picture, we defined several parameters of the air gap: $R_{f}, R_{b}$, and $d$, as shown in Fig. 4 (b). $R_{f}$ is the height, $R_{b}$ is the sidewall curvature, and $d$ is the width. The relationship between these three parameters can be expressed as

$$
R_{b}=\left(R_{f}^{2}+\frac{d^{2}}{4}\right) / d
$$

We model the micro-air-gap-based IFPI sensor as a paraxial optical system. Two fundamental matrices are useful in the analysis:

(1) Transmission matrix in air $M_{\text {Trans }}$,

$$
M_{\text {Trans }}=\left[\begin{array}{ll}
1 & d \\
0 & 1
\end{array}\right]
$$

where $d$ is the transmission distance.

(2) Refraction matrix at a spherical boundary $M_{\text {refr }}{ }^{9}$ :

$$
M_{\text {refr }}=\left[\begin{array}{cc}
1 & 0 \\
-\left(n_{2}-n_{1}\right) / n_{2} R & n_{1} / n_{2}
\end{array}\right],
$$

where $n_{2}$ and $n_{1}$ are refractive indices of the media, and $R$ is the radius of the curvature.

For a micro-air-gap-based IFPI sensor, the light propagates from the left spherical surface of the air gap with a radius of $R_{b}$, then transmits a distance of $d$ in air, and passes through the right spherical sur-

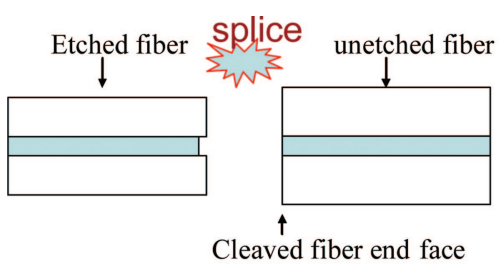

(a)

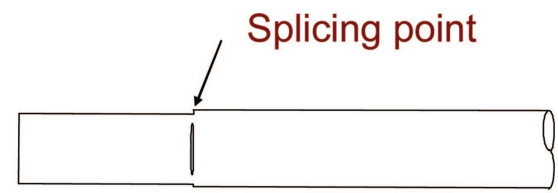

(b)
Fig. 2. (Color online) Fusion splice (a) before splicing, (b) after splicing. 


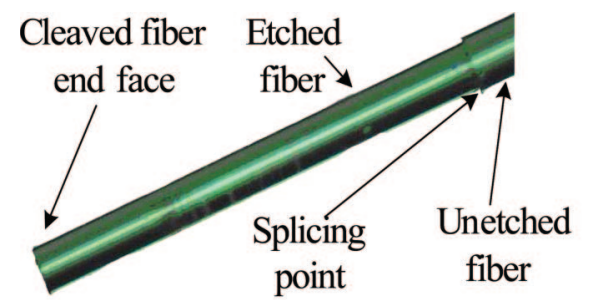

(a)

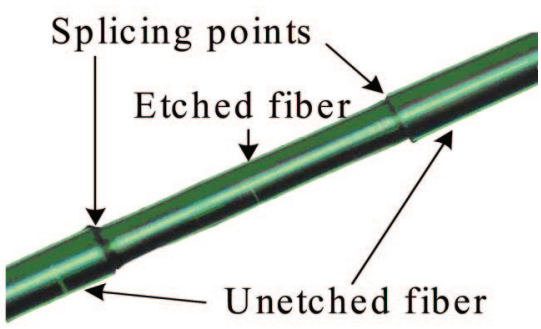

(b)
Fig. 3. (Color online) Two IFPI structures. face. The $2 \times 2$ matrix of the system can be given by

$$
M=M_{\text {refr_right }} M_{\text {Trans }} M_{\text {refr_left }} .
$$

The scalar field of the fundamental linearly polarized $\left(\mathrm{LP}_{01}\right)$ mode in a weakly guiding, step-index, circular-core optical fiber can be approximated by a Gaussian distribution. A complex curvature parameter $q(z)$ can be used to fully describe the input and output Gaussian beams ${ }^{9}$

$$
\frac{1}{q(z)}=\frac{1}{R_{w}(z)}-j \frac{\lambda}{\pi W^{2}(z)},
$$

where real parameter $W(z)$ is the beam waist, real parameter $R_{w}(z)$ is the wavefront curvature, and $\lambda$ is the wavelength.

The propagation of a Gaussian beam can be determined by the well-known $A B C D$ rule. ${ }^{10}$ When a Gaussian beam passes through an optical system with a matrix

$$
M=\left[\begin{array}{ll}
A & B \\
C & D
\end{array}\right]
$$

the output beam waist and wavefront curvature of the Gaussian beam are subject to changes and related to the input Gaussian beam by ${ }^{9,11}$

$$
q_{\text {out }}(z)=\frac{A q_{\text {in }}(z)+B}{C q_{\text {in }}(z)+D},
$$

where $q_{\text {out }}(z)$ and $q_{\text {in }}(z)$ describe the input and output Gaussian beams, respectively.

The power transmission coefficient $T$ of the air gap can be calculated by using input and output Gaussian beam waists $^{10,12}$ :

$$
T=\left(\frac{2 W_{\text {in }} W_{\text {out }}}{{W_{\text {in }}^{2}+W_{\text {out }}^{2}}^{2}}\right)^{2} .
$$

Before splicing, we obtained the approximation from Fig. 1(c) that the base diameter of the etched pit on the fiber end face is $16 \mu \mathrm{m}$. A little expansion of this diameter is expected due to splicing so $18 \mu \mathrm{m}$ is used for $R_{f}$. The possible shapes of the air gap are shown in Fig. 5(a). Substituting Eqs. (1)-(6) into Eq. (7), the transmission coefficient of the air gap with different widths but fixed height $R_{f}$ [shown in Fig. 5(a)] can be calculated and is shown in Fig. 6. The smaller the value of $d$, the higher the coupling efficiency of the air gap.

The power transmission coefficient is high for small $d$. If we assume that $d$ equals $1.5 \mu \mathrm{m}$ and change the value of $R_{f}$, the possible shape of the air gap is shown in Fig. 5(b). The power transmission coefficient versus $R_{f}$ is shown in Fig. 7 . As long as $R_{f}$ is greater than $10 \mu \mathrm{m}$, the power transmission coefficient will maintain at a value that is not sensitive to the value of $R_{f}$.

In the sensor fabrication, we control the wet chemical etching to obtain a proper value of $R_{f}$, and control the fusion-splice process to obtain a small value of $d$. To obtain the highest coupling efficiency, we should fabricate the sensors with an air gap of $d<3 \mu \mathrm{m}$ and $R_{f}>10 \mu \mathrm{m}$.

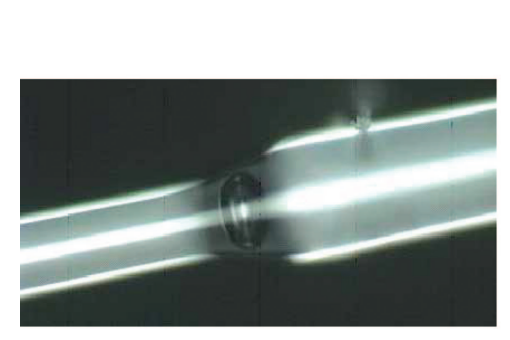

(a)

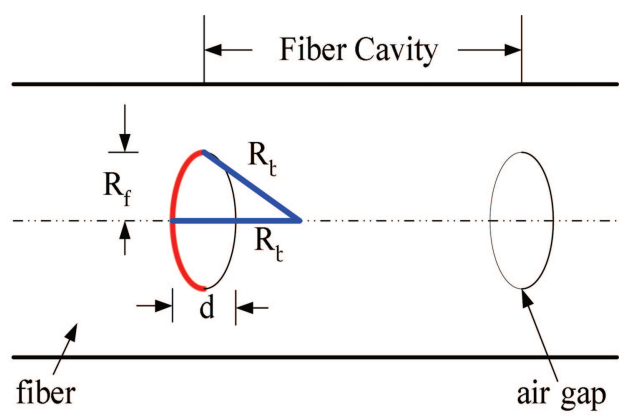

(b)
Fig. 4. (Color online) Schematic of the sensor. 
10
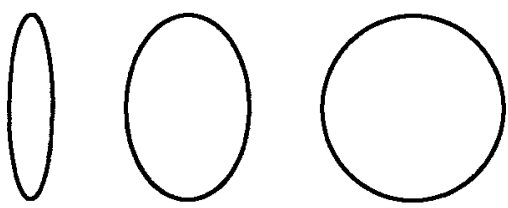

(a)

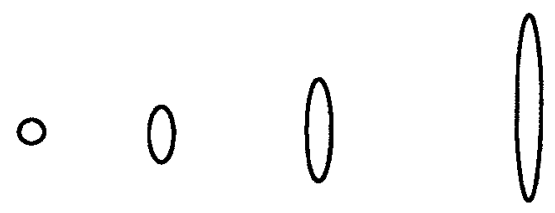

(b)

Fig. 5. Possible shapes of the air gap, (a) when $R_{f}$ is a fixed value; (b) when $d$ is a fixed value.

When $R_{f} \gg d$ and $L \gg d$, the interference spectrum can be analyzed by assuming that the spherical surface of the air gap is a plane surface, and the air gap is a single reflector. The model of one air-gap interferometer is shown in Fig. 8, where $R_{1}, R_{2}$ and $\eta_{1}, \eta_{2}$ are the reflectance and coupling coefficients of the air gap and the fiber end face. The model in Fig. 8 ignores the $\pi / 2$ phase change induced by reflectors. The reflectance of an air gap $\left(R_{1}\right)$ is measured to be in the range of -13 to $-16 \mathrm{~dB}$ at a wavelength of $1550 \mathrm{~nm}$ depending on the splicer parameters used.

By summing all the reflected fields from the air gap, the total field received by the detector is given by

$$
E=E_{0}\left[R_{1}+\eta_{1}^{2}\left(1-R_{1}\right)^{2} R_{2} e^{-j 2 \varphi}\right],
$$

assuming that $R_{1}$ and $R_{2}$ are very small, and the FP interferometer can be approximated to a two-beam or a Fizeau interferometer with all the multipath reflections neglected.

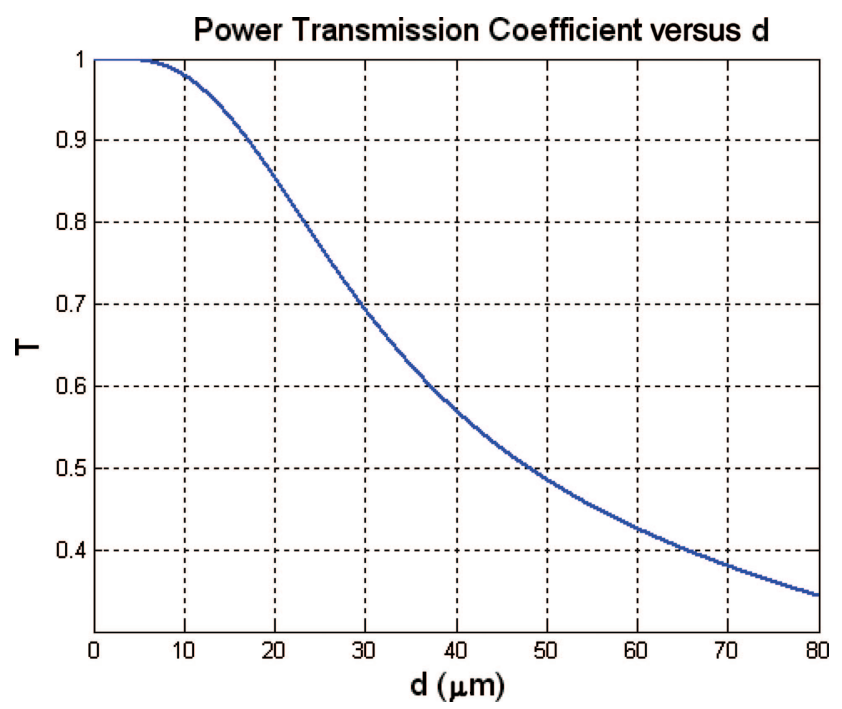

Fig. 6. (Color online) Power transmission coefficient when $R_{f}$ is fixed.

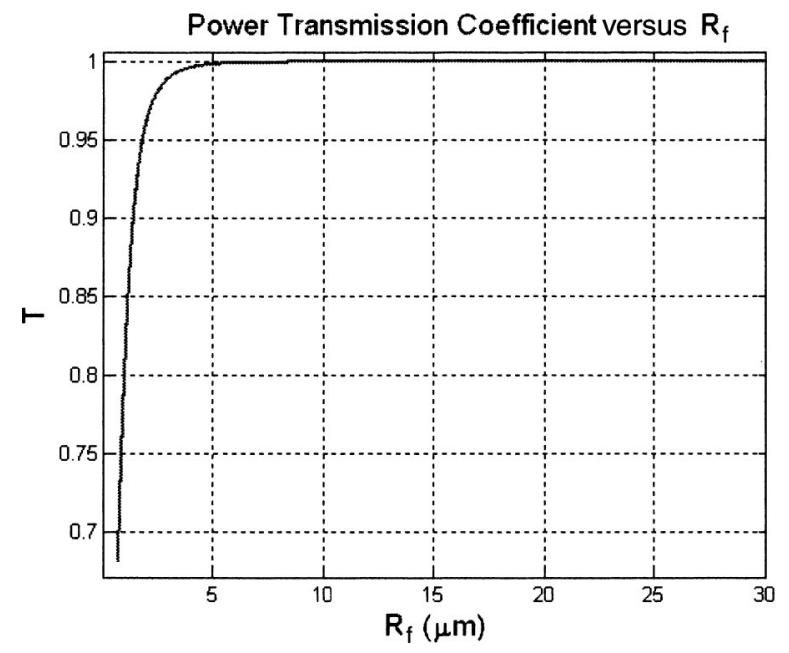

Fig. 7. Power transmission coefficient when $d$ is fixed.

The phase shift between two successive reflections is

$$
\varphi=\frac{4 \pi n L}{\lambda},
$$

where $L$ is the fiber cavity length and $n(n=1.4682$ for Corning SMF-28 at a $1550 \mathrm{~nm}$ wavelength) is the refractive index of the fiber. The intensity $I$ is, thus,

$$
\begin{aligned}
I= & E \times E^{*} \\
= & E_{0}{ }^{2} R_{1}{ }^{2}+R_{2}{ }^{2}\left(1-R_{1}\right)^{4} \eta_{1}{ }^{4}+2 R_{1} R_{2}\left(1-R_{1}\right)^{2} \\
& \times \eta_{1}{ }^{2} \cos (2 \varphi) .
\end{aligned}
$$

In Fig. 9, the solid curve represents the spectrum captured from a real sensor, and the dashed curve is the calculation result from Eq. (10) when we set $L=63.58 \mu \mathrm{m}, R_{1}=-15.23 \mathrm{~dB}$, and $R_{2}=-14.00$ $\mathrm{dB}$. Since the air-gap width $d$ is small as expected, the low-frequency envelope caused by the air gap cannot be observed in the spectral range of measurement from 1520 to $1570 \mathrm{~nm}$.

\section{Experimental Results}

Two structures of the micro-air-gap-based IFPI sensors could be used in a wide variety of applications. In this section, we present temperature measurement by using the single micro-air-gap structure [shown in Fig. 3(a)] and strain measurement by using the double micro-air-gap structure [shown in Fig. 3(b)].

A real-time-component testing system (RT-CTS) developed by Micron Optics, Incorporated was used

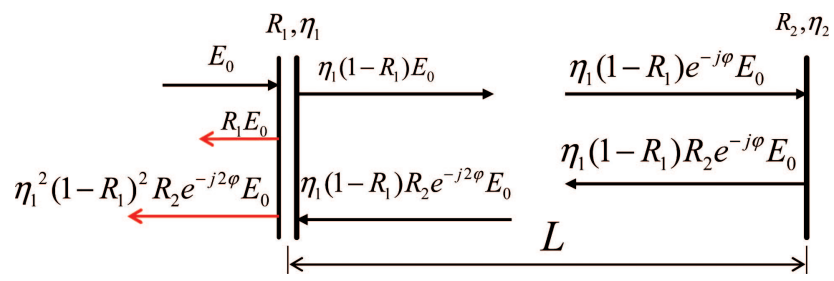

Fig. 8. (Color online) Multiple-reflection model. 


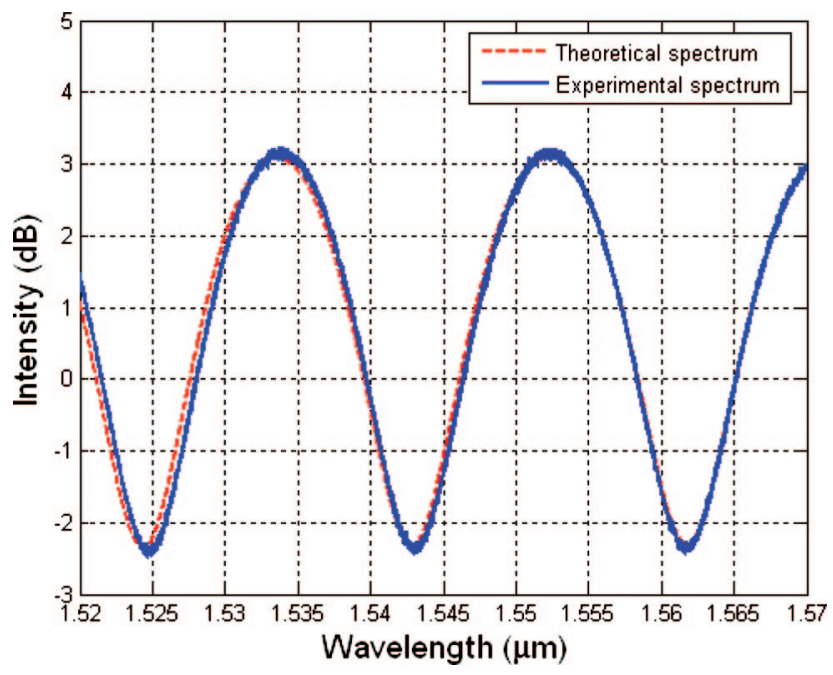

Fig. 9. (Color online) Spectrum comparison between experiment and theory.

to monitor the reflection spectrum (shown in Fig. 9). The principal diagram of the experiment setup is shown in Fig. 10. Particular signal processing software developed by CPT, Virginia Tech, provides the capacity to trace the peaks of the reflection spectrum and calculate the optical path difference (OPD) of the sensor. ${ }^{13,14}$ First, the rough value of OPD is calculated through wavelengths of two adjacent peaks in the interference spectrum by

$$
\mathrm{OPD}_{\text {rough }}=\frac{\lambda_{1} \lambda_{2}}{2\left(\lambda_{1}-\lambda_{2}\right)}
$$

where $\lambda_{1}$ and $\lambda_{2}$ represent the wavelengths at two adjacent peak points. OPD $\mathrm{O}_{\text {rough }}$ is then used to improve the accuracy of $K_{m}$ in Eq. (12), and a more accurate OPD can then be obtained by

$$
\mathrm{OPD}=\frac{\left(m 2 \pi-\varphi_{0}\right) \lambda_{m}}{4 \pi}=\frac{K_{m}}{2} \lambda_{m}
$$

where $\lambda_{m}$ is the wavelength of the $m$ th peak and $K_{m}$ is called the $m$ th-order number of the spectrum. $\varphi_{0}$ is the arbitrary initial phase difference of interference

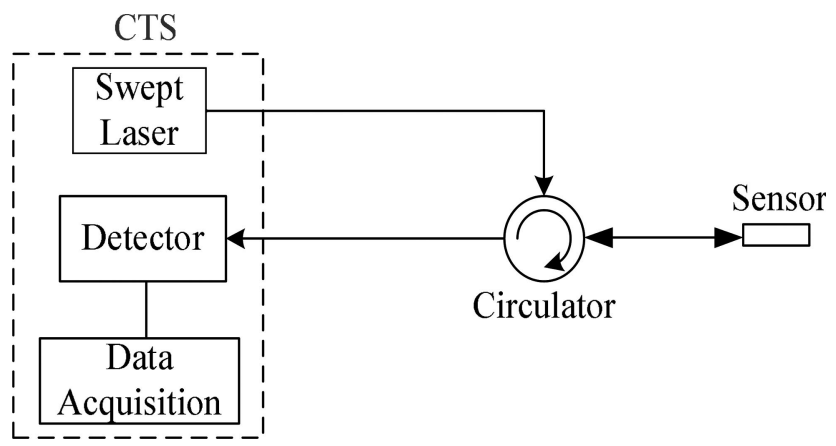

Fig. 10. Schematic of the sensor system.

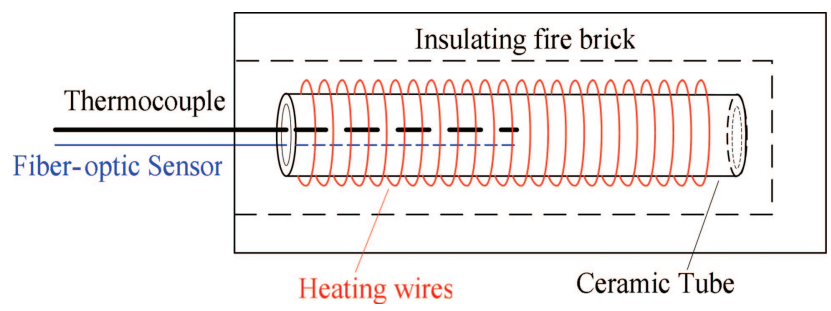

Fig. 11. (Color online) Temperature measurement setup.

signals. The system offers the measurement range from 1520 to $1570 \mathrm{~nm}$ with $1 \mathrm{pm}$ accuracy.

\section{A. Temperature Measurement with Single Micro-Air-Gap Structure}

The performance of a single micro-air-gap sensor for temperature measurement was demonstrated from room temperature $\left(23{ }^{\circ} \mathrm{C}\right)$ to $700{ }^{\circ} \mathrm{C}$. The sensor, along with a type $\mathrm{K}$ thermocouple (Omega, Incorporated), was inserted in a ceramic tube (inner diameter is $0.25 \mathrm{in}$.) and placed in the self-made furnace shown in Fig. 11. The reflection spectrum was recorded every $1 \mathrm{~min}$. The change in the OPD of the sensor with respect to the temperature is plotted in Fig. 12. Data was recorded every $50{ }^{\circ} \mathrm{C}$ for three runs. The total OPD change corresponding to the temperature change from $50{ }^{\circ} \mathrm{C}$ to $700{ }^{\circ} \mathrm{C}$ is $2.9 \mu \mathrm{m}$. Comparing the measurement with the thermocouple, the calibration accuracy of the sensor can be obtained. The temperature difference between the optical sensor and that of the thermocouple is $\pm 1.9^{\circ} \mathrm{C}$ corresponding to a calibration accuracy of $\pm 0.28 \%$ of full scale, as shown in Fig. 13. The standard deviation of this sensor tested for $24 \mathrm{~h}$ at room temperature is $0.27 \mathrm{~nm}\left(0.06{ }^{\circ} \mathrm{C}\right)$. Thus the resolution of the system is approximately $0.54 \mathrm{~nm}\left(0.12^{\circ} \mathrm{C}\right)$, which corresponds to $0.03 \%$ of full scale.

\section{B. Strain Measurement With the Double Micro-Air-Gap Structure}

The multiplexed strain measurement with the double micro-air-gap fiber sensor is also demonstrated using

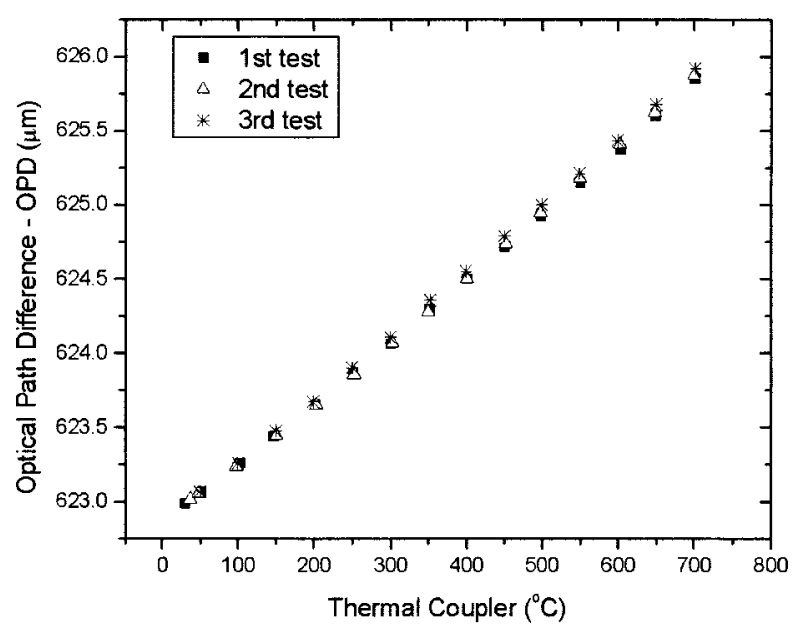

Fig. 12. Temperature measurement results. 


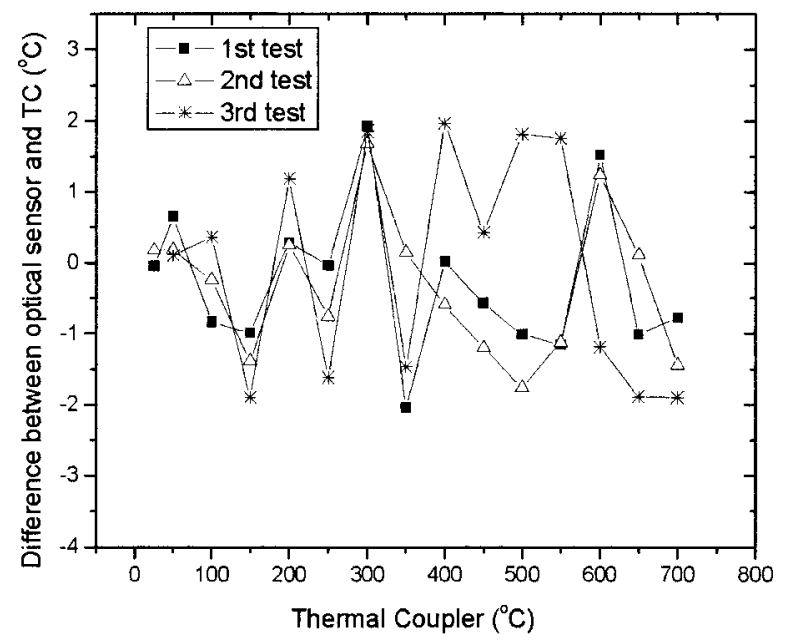

Fig. 13. Difference between the readouts of the optical sensor and the thermocouple.

a cantilever beam configuration shown in Fig. 14 . Two optical sensors with different cavity lengths are connected serially along a single fiber and surface bounded on the cantilever beam to measure strains at two points. Strain induced within two sensors due to the beam deflection can be calculated by

$$
\varepsilon_{1,2}=\frac{3 D_{1,2} t \delta}{2 D^{3}}
$$

where $t=2.54 \mathrm{~mm}$ is the beam thickness, $D=$ $308.85 \mathrm{~mm}$ is the beam length, $D_{1}=239.25 \mathrm{~mm}$ and $D_{2}=98.13 \mathrm{~mm}$ are the distances between the beam ends, where sensors 1 and 2 were bounded, and $\delta$ is the beam deflection at the free end. The OPD change of optical sensor due to strain is determined by the dimension change of the fiber sensor and the elastooptic effect in the $\mathrm{SiO}_{2}$ fiber. The normalized OPD change can be given as

$$
\frac{\Delta \mathrm{OPD}}{\mathrm{OPD}}=\left(1-P_{e}\right) \varepsilon,
$$

where $P_{e}$ is the effective elasto-optic coefficient, and $\varepsilon$ is the strain applied on the sensor.

When the optical length of the fiber section between two sensors is longer than the coherent length of the light source, the total reflectivity is a linear superposition of two sinusoidal signals with different frequencies corresponding to different optical sensor cavity lengths. Two Hamming-windowed far-infrared (FIR) bandpass filters were applied on the fast Fou-

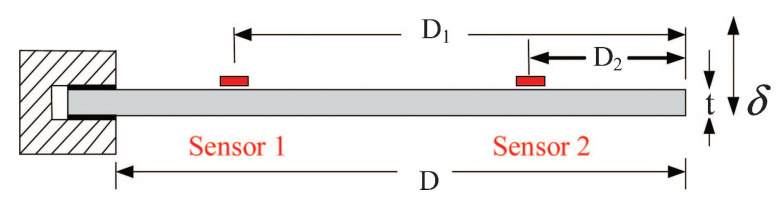

Fig. 14. (Color online) Strain measurement setup.

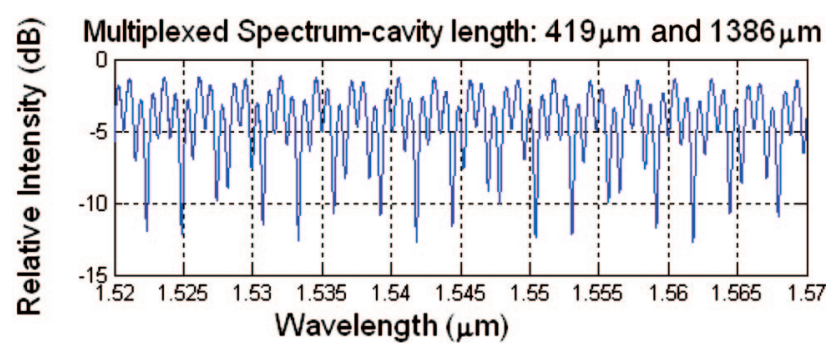

(a)

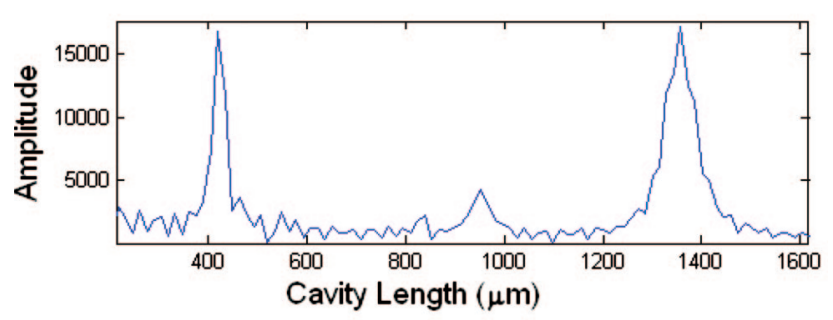

(b)

Fig. 15. (Color online) (a) Spectrum and (b) results of the FFT of a multiplexed sensor.

rier transform (FFT) of the total reflection spectrum, and the reflection spectrum of each sensor can be obtained separately from which OPDs of both sensors are calculated. ${ }^{14}$ The fiber cavity lengths of two sensors are 419 and $1386 \mu \mathrm{m}$. The spectra of the two IFPI sensors captured by the CTS and its FFT are shown in Fig. 15.

During the experiment, the beam end deflection was varied from -22 to $20 \mathrm{~mm}$ with a step of $2 \mathrm{~mm}$. Strain from -700 to $600 \mu \varepsilon$ and from $-300 \mu \varepsilon$ to $250 \mu \varepsilon$ was induced in sensors 1 and 2 , respectively. At each strain level, ten OPDs were calculated for each sensor and averaged. The experimental result is shown in Fig. 16 in which the normalized OPD change of optical sensors versus theoretical strain calculated via Eq. (13) was plotted. The optical sensor's sensitivity can be estimated by taking the slope of the linear fitting of the data points. A sensitivity of

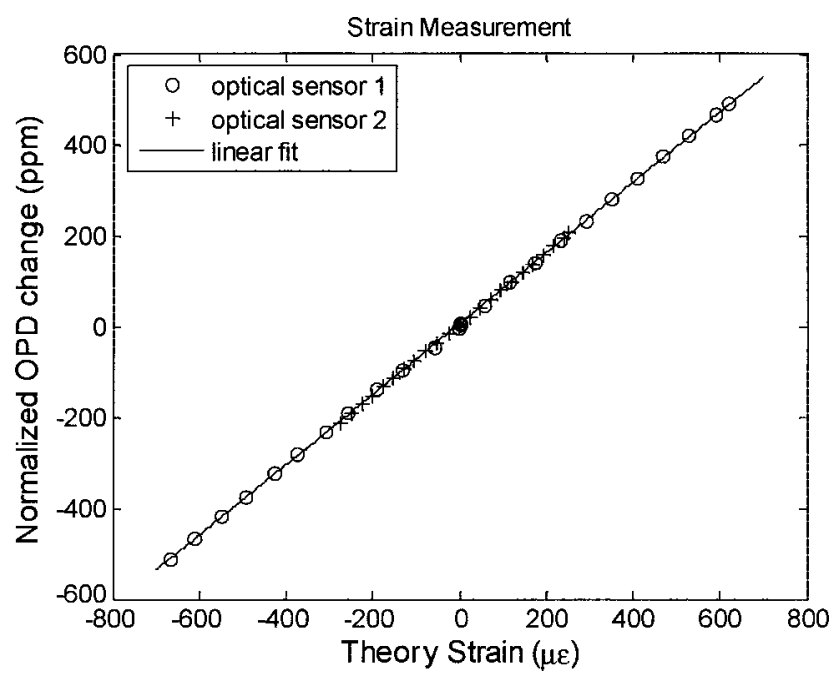

Fig. 16. Strain measurement results by optical sensors. 


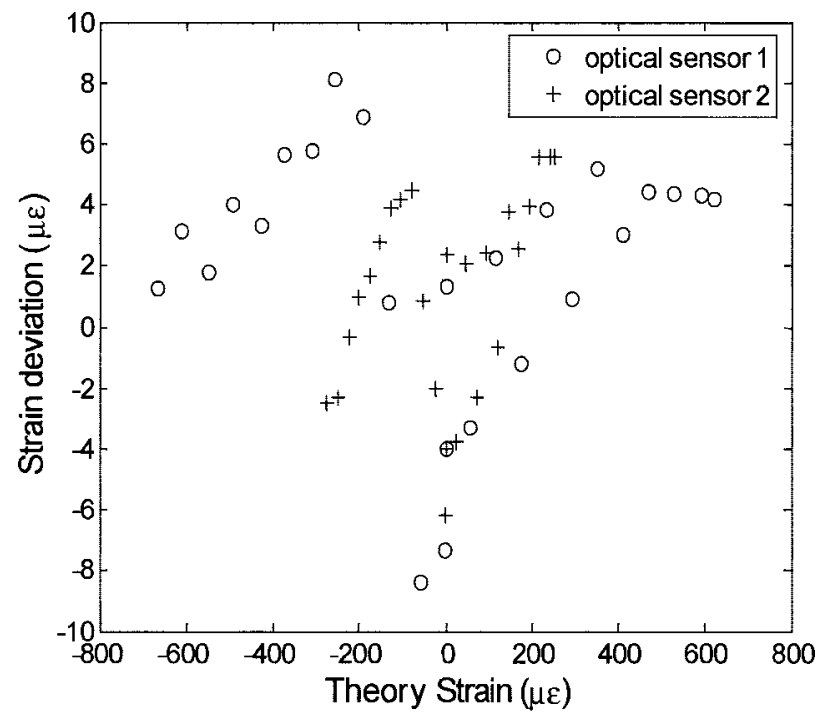

Fig. 17. Standard deviation of strain measurement.

0.777 parts per million/ $\mu \varepsilon$ was obtained. The standard deviation of the strain measured by optical sensor and the theoretical strain was plotted in Fig. 17. The maximum deviation of $\pm 9 \mu \varepsilon$ corresponding to an accuracy of $\pm 1.5 \%$ system internal accuracy was observed.

\section{Conclusions}

In this paper, a novel method for fabricating IFPI sensors is presented and analyzed. Sensors with two slightly different structures are fabricated by carefully controlling the air-gap size to obtain high reflectance and low transmission loss. The first structure with only one micro-air gap and a cleaved fiber end is simpler and better for a single point measurement, while the second structure is more suitable for multiple point measurements via sensor multiplexing. The second structure can also be connected with other fiber-optic sensors, such as long period grating, EFPI sensors, and other IFPI sensors for temperature compensated measurement. The resolution of $0.12{ }^{\circ} \mathrm{C}$ for the temperature measurement and $0.767 \times 10^{-6}$ per microstrain for the stain measurement can be achieved. More applications, such as thin-film thickness measurement for biosensing, are under investigation.
The work presented is supported by the U.S. Department of Energy under grant DE-FC36-01G011050. The authors thank the partial donation of RT-CTS by Micron Optics.

\section{References}

1. C. E. Lee and H. F. Taylor, "Optical-fiber Fabry-Perot embedded sensor," Opt. Lett. 14, 1225-1227 (1991).

2. H. Singh and J. S. Sirkis, "Simultaneously measuring temperature and strain using optical fiber microcavities." J. Lightwave Technol. 15, 647-653 (1997).

3. W.-H. Tsai and C.-J. Lin, "A novel structure for the intrinsic Fabry-Perot fiber-optic temperature sensor." J. Lightwave Technol. 19, 682-686 (2001).

4. F. Shen, W. Peng, K. Cooper, G. Pickrell, and A. Wang, "UVinduced intrinsic Fabry-Perot interferometric fiber sensors," in Sensors for Harsh Environments, A. Wang, ed., Proc. SPIE 5590, 47-56 (2004).

5. A. Klini, D. E. Bourillot, S. Emonin, P. Papadopoulos, J. P. Goudonnet, and G. Kotrotsios, "Reproducible optical fiber tips for photon scanning tunneling microscopy with very small $\left(<5^{\circ}\right)$ cone angle," J. Lightwave Technol. 16, 1220-1227 (1998).

6. A. Sayah, C. Philipona, P. Lambelet, M. Pfeffer, and F. Marquis-Weible, "Fiber tips for scanning near-field optical microscopy fabricated by normal and reverse etching," Ultramicroscopy 72, 59 (1998).

7. S. I. Hosain, Y. Lacroute, and J. P. Goudonnet, "A simple low-cost highly reproducible method of fabricating optical fiber tips for a photon scanning tunneling microscope," Microwave Opt. Technol. Lett. 13, 243-245 (1996).

8. X. Chen, F. Shen, Y. Zhang, Z. Wang, and A. Wang, "A novel Fabry-Perot fiber-optic sensor with multiple applications," in Sensors for Harsh Environments, A. Wang, ed., Proc. SPIE 5590, 111-121 (2004).

9. R. C. Gauthier, M. Friesen, T. Gerrard, W. Hassouneh, P. Koziorowski, D. Moore, K. Oprea, and S. Uttamalingam, "Selfcentering of a ball lens by laser trapping: fiber-ball-fiber coupling analysis," Appl. Opt. 42, 1610-1619 (2003).

10. A. Gerrard and J. M. Burch, Introduction to Matrix Methods in Optics (Wiley, 1975), p. 119.

11. N. Kashima, Passive Optical Components for Optical Fiber Transmission (Artech House, 1995), p. 59.

12. D. Marcuese, "Loss analysis of single-mode fiber splices," Bell Syst. Tech. J. 56, 703-717 (1977).

13. B. Qi, G. Pickrell, J. Xu, P. Zhang, Y. Duan, W. Peng, Z. Huang, W. Hou, H. Xiao, R. G. May, and A. Wang, "Novel data processing techniques for dispersive white light interferometer," Opt. Eng. 42, 3165-3171 (2003).

14. F. Shen and A. Wang, "Frequency-estimation-based signalprocessing algorithm for white-light optical fiber Fabry-Perot interferometers," Appl. Opt. 44, 5206-5214 (2005). 